\title{
OC32 - Nursing interventions for empowering parents of premature infants before discharge from the neonatal intensive care unit: a scoping review
}

\author{
Denise Araújo (Portugal) ${ }^{1}$; Maria do Céu Barbieri-Figueiredo \\ $(\text { Portugal })^{2}$
}

${ }^{1}$ ICBAS, Universidade do Porto; ${ }^{2}$ Escola Superior de Enfermagem do Porto

Theme: Complex health care and chronic disease management.

Keywords: Discharge, family, nursing, premature infant, transitional care, nursing.

Family-centred nursing interventions in perinatal period are of utmost importance for empowering parents and preparing the discharge from neonatal intensive care unit. The purpose of this scoping review was to summarize the available evidence related to familycentred nursing interventions during hospitalization in perinatal period, of infants born with less than 37 weeks of gestational age, for empowering parents before discharge from hospital.

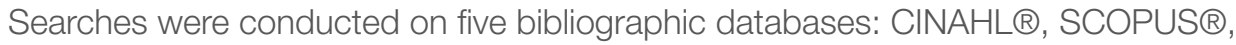
MEDLINE®, MEDLACTINA®, Cochrane Central Register of Controlled Trials $®$. A total of 15 studies, published between 2004 and 2015, were included in the review. Nurses considered the following issues as essential for discharge preparation and empowerment of families: availability of social aids for vulnerable families, 24-hour telephone access to the neonatal units, tailored educational materials and group sessions, continuing education for staff and community-based support to parents post-discharge.

\section{OC33 - Young people's eating behaviours in Vila Nova de Famalicão}

\author{
Ilda Fernandes (Portugal) ${ }^{1}$; Manuela Martins (Portugal) ${ }^{1}$; \\ Luísa Andrade (Portugal) ${ }^{1}$; Sara Soares (Portugal) ${ }^{1}$
}

${ }^{1}$ ESEP

Theme: Nutrition

Keywords: Adolescent, eating, health behaviours, social determinants.

Overweight and obesity are prevalent health problems among young people, with implications on wellbeing and health in adult life. A quantitative, descriptive and transversal study was performed to analyse the eating behaviours perception of adolescents from a school community in Vila Nova de Famalicão. A self-administered questionnaire was applied to 1,614 adolescents attending secondary and higher education in the academic year 2013/14, aged between 14-21 years.

From the total participants, 56.9\% were female and 86.5\% attended secondary school. Results showed that $85.3 \%$ ate a variety of food, including animal origin products; $32.1 \%$ 